\title{
Comparative analysis of impact of human occupancy on indoor microbiomes
}

\author{
Liu Cao ${ }^{1}$, Lu Yang ${ }^{1}$, Clifford S. Swanson ${ }^{1}$, Shuai Li $\left.(\bowtie)\right)^{1}$, Qiang He $\left.(\bowtie)\right)^{1,2}$ \\ 1 Department of Civil and Environmental Engineering, The University of Tennessee, Knoxville, TN 37996, USA \\ 2 Institute for a Secure and Sustainable Environment, The University of Tennessee, Knoxville, TN 37996, USA
}

\section{H I G H L I G H T S}

- Exposure to indoor microbiomes is a public health concern in educational facilities.

- Indoor microbiomes were characterized in two multifunctional university buildings.

- Human occupancy had significant impact on the composition of indoor microbiomes.

- The skin microbiota of occupants represented important sources of indoor microbiomes.

\section{A R T I C L E I N F O}

Article history:

Received 30 July 2020

Revised 20 October 2020

Accepted 23 October 2020

Available online 10 December 2020

\section{Keywords:}

Built environment

Indoor microbiome

Occupant

Building

Sequencing

\section{GRAPHIC ABSTRACT}
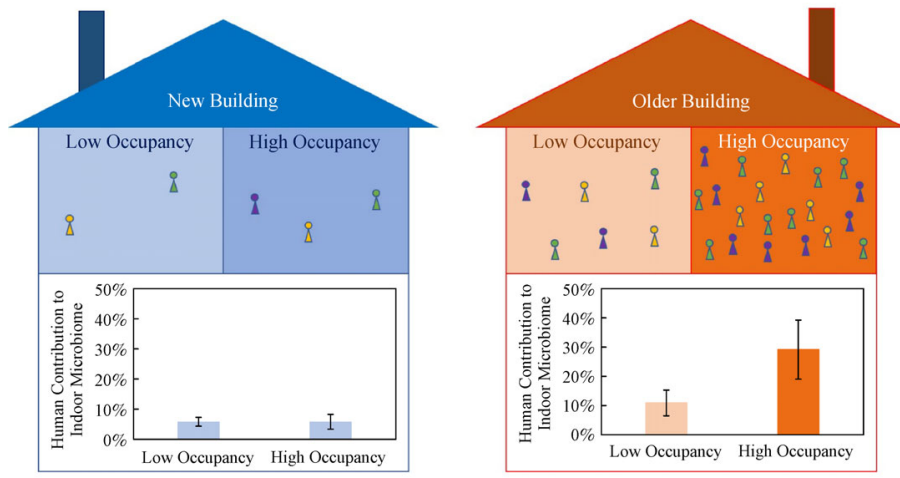

\section{A B S T R A C T}

Educational facilities serve as community hubs and consequently hotspots for exposure to pathogenic microorganisms. Therefore, it is of critical importance to understand processes shaping the indoor microbiomes in educational facilities to protect public health by reducing potential exposure risks of students and the broader community. In this study, the indoor surface bacterial microbiomes were characterized in two multifunctional university buildings with contrasting levels of human occupancy, of which one was recently constructed with minimal human occupancy while the other had been in full operation for six years. Higher levels of human occupancy in the older building were shown to result in greater microbial abundance in the indoor environment and greater proportion of the indoor surface bacterial microbiomes contributed from human-associated microbiota, particularly the skin microbiota. It was further revealed that human-associated microbiota had greater influence on the indoor surface bacterial microbiomes in areas of high occupancy than areas of low occupancy. Consistent with minimal impact from human occupancy in a new construction, the indoor microbiomes in the new building exhibited significantly lower influence from human-associated microbiota than in the older building, with microbial taxa originating from soil and plants representing the dominant constituents of the indoor surface bacterial microbiomes. In contrast, microbial taxa in the older building with extensive human occupancy were represented by constituents of the human microbiota, likely from occupants. These findings provide insights into processes shaping the indoor microbiomes which will aid the development of effective strategies to control microbial exposure risks of occupants in educational facilities.

(C) Higher Education Press 2020

\section{Introduction}

It has been estimated that more than $85 \%$ of human activities occur in enclosed buildings (Klepeis et al., 2001;

$凶$ Corresponding authors

E-mail: sli48@utk.edu (S. Li); qianghe@utk.edu (Q.He)
Schweizer et al., 2007). Evidently, the built environment has a significant impact on the wellbeing of humans. Given that microorganisms are closely associated with human health, exposure to the microbial populations inhabiting the built environment, i.e. the indoor microbiome, has been linked to various health outcomes of the occupants, such as the regulation of immune functions (Lax et al., 2015), development of allergic diseases (Sbihi et al., 2019), 
spread of antibiotics resistance (Ben Maamar et al., 2020; Song et al., 2020), and particularly transmission of pathogens (Lax and Gilbert, 2015) which is an urgent concern due to the rapid spread of severe acute respiratory syndrome coronavirus 2 (SARS-Cov-2) in indoor environments (Allen and Marr, 2020).

To develop effective management strategies for the built environment to protect public health, indoor microbiomes have been characterized in a variety of built environments, including residential homes, healthcare facilities, office buildings, retail stores, public restrooms, and transit systems (Adams et al., 2015). However, there remains a dearth of studies focusing on the indoor microbiome in educational facilities, which typically serve as community hubs and subsequently hotspots for pathogen exposure. Indeed, more than 76 million students are enrolled in schools and colleges in the US alone (US Census Bureau, 2018). The mass flux of students, parents, and other community members are directly or indirectly exposed to the indoor microbiomes in these educational facilities. It is of critical importance to gain a systematic understanding of the indoor microbiomes in these educational facilities, which are frequently densely-populated with high risks of microbial exposure, for the development of effective control and management practices to reduce exposure risks.

Therefore, the main objective of this study was to characterize the indoor microbiomes in two multifunctional university buildings, of which one was a new construction while the other had been in use for six years. It was found that human occupancy had contributed to the marked contrasts between the indoor microbiomes in the two buildings, providing valuable insights into the processes shaping the indoor microbiomes in educational facilities.

\section{Materials and methods}

\subsection{Sample collection}

Two multifunctional buildings, Buildings $\mathrm{T}$ and $\mathrm{M}$, on a university campus were selected for sampling. The two buildings shared similarities in function, management practice, and layout, having classrooms, graduate student offices, faculty offices, administrative offices, research laboratories, and student lounges. The two buildings were controlled by identical HVAC systems with the room temperature set at $72^{\circ} \mathrm{F}$ and relative humidity levels between $40 \%$ and $60 \%$ during the month of August when sampling occurred. However, the two buildings differed in the level of human occupancy. Building $\mathrm{M}$ was recently constructed with minimal human occupancy at the time of sampling. In contrast, Building $\mathrm{T}$ had been in use for six years when sampled.

A total of 20-five desk surfaces were selected for sampling in each building according to the level of human occupancy. Rooms with seating capacity less than 0.25 persons $/ \mathrm{m}^{2}$ were classified as low-occupancy areas, which included offices and research laboratories. In contrast, rooms with seating capacity greater than 0.50 persons $/ \mathrm{m}^{2}$ were classified as high-occupancy areas, which included classrooms. In addition, lobbies, located at the main entrances of the buildings, were also classified as highoccupancy areas due to high traffic of occupants. Sampling was conducted simultaneously in both buildings during the lunch break (12:00-1:00 PM) when classes were dismissed, which was intended to minimize interferences from occupant traffic during sampling. Two samples were collected from each of the 20-five surface sampling sites, one for heterotrophic plate count (HPC) and the other for 16S rRNA gene amplicon sequencing. Individually packed sterile Puritan polyester-tipped swabs (Puritan, Guilford, Maine, USA) were used to collect both samples. The swabs were pre-moistened with sterile phosphate-buffered saline-tween (PBST, $\mathrm{pH}=7.2$ ) solution, pressed against the side of the PBST container to remove excess liquid, then pressed onto the surface, and moved back and forth to cover a $2.25-\mathrm{dm}^{2}$ surface area using previously described protocols (Rose et al., 2004). Sample collection was completed within four hours for both buildings. Following surface sampling, one swab was preserved in $50 \mathrm{~mL}$ of PBST for HPC analysis within $6 \mathrm{~h}$ of sampling. The second swab was preserved at $-20^{\circ} \mathrm{C}$ until DNA extraction for amplicon sequencing.

\subsection{Heterotrophic plate count (HPC) analysis}

HPC analysis was used as a measure of the abundance of heterotrophic bacterial populations in the surface samples. The HPC agar plates containing the solid growth medium were prepared one day prior to sampling according to established standard procedures (Reasoner, 2004). The agar plates were made by first preparing a liquid medium containing (per liter) $5.0 \mathrm{~g}$ tryptone, $2.5 \mathrm{~g}$ yeast extract, 1.0 g glucose, and $15.0 \mathrm{~g}$ agar. After autoclaving at $121^{\circ} \mathrm{C}$ for $30 \mathrm{~min}$, the medium was poured into sterile plates to a thickness of 1-2 $\mathrm{mm}$ and cooled at room temperature to solidify. The agar plates were then sealed with parafilm and stored at $4^{\circ} \mathrm{C}$ before use.

Swab samples kept in $50 \mathrm{~mL}$ of PBST were vortexed for $5 \mathrm{~min}$ and the resulting suspensions were filtered through $0.22 \mu \mathrm{m}$ (pore size) Isopore polycarbonate membrane filters (Merck KGaA, Darmstadt, Germany). The filters were then placed onto pre-prepared HPC agar plates and incubated for $48 \mathrm{~h}$ before the colonies were counted. Results from HPC analysis were shown in CFU $/ \mathrm{dm}^{3}$ which was normalized by the sampling area.

\subsection{DNA extraction, amplification and sequencing}

DNA was extracted directly from each swab sample using 
the FastDNA Spin Kit for Soil (MP Biomedicals, Irvine, California, USA) according to the manufacturer's instructions. The DNA extracts were then purified with the Genomic DNA Clean \& Concentrator ${ }^{\mathrm{TM}}-10$ kit (Zymo Research, Irvine, California, USA) following the manufacturer's instructions. DNA concentrations were determined using the NanoDrop ND-3300 fluorospectrometer (Thermo Scientific, Waltham, Massachusetts, USA).

Purified DNA extracts were amplified targeting the V4 region of the $16 \mathrm{~S}$ rRNA gene with primers $515 \mathrm{~F}$ (GTGCCAGCMGCCGCGGTAA) and 806R (GGACTACHVGGGTWTCTAAT) as previously described (Wyckoff et al., 2017). The polymerase chain reaction (PCR) was prepared with a cocktail mix containing 12.5 $\mu$ L Phusion Flash High-Fidelity PCR Master Mix (Thermo Scientific, Waltham, Massachusetts, USA), $10 \mu \mathrm{L}$ ultrapure water, $1 \mu \mathrm{L}$ forward primer, $1 \mu \mathrm{L}$ reverse primer, and $2 \mu \mathrm{L}$ (100 to $150 \mathrm{ng}$ ) DNA template. The PCR program included one cycle at $94^{\circ} \mathrm{C}$ for $3 \mathrm{~min}$, followed by 35 cycles at $94^{\circ} \mathrm{C}$ for $45 \mathrm{~s}, 55^{\circ} \mathrm{C}$ for $60 \mathrm{~s}$, and $72^{\circ} \mathrm{C}$ for $90 \mathrm{~s}$, with a final extension at $72^{\circ} \mathrm{C}$ for $10 \mathrm{~min}$. PCR products were examined by an Agilent 2100 Bioanalyzer instruments with Agilent DNA 7500 kits (Santa Clara, California, USA) for amplicon quantification and quality verification.

Subsequently, amplicons were pooled and amplicon library concentrations were measured using the KAPA Illumina Library Quantification Kit (Kapa Biosystems, Wilmington, Massachusetts, USA). The PhiX control library (Illumina, San Diego, California, USA) was added to the amplicon libraries to increase diversity. Sequencing of the amplicon libraries was performed with an Illumina MiSeq System (Illumina, San Diego, California, USA) at the University of Tennessee Genomics Core Facilities.

\subsection{Data processing}

Raw amplicon sequences were processed using the CLC Genomics Workbench 10 (Qiagen, Aarhus, Denmark) with the microbial genomics module following previously described protocols (Treu et al., 2018). Briefly, forward and reverse sequence reads were aligned and sequence pairs with more than 3 mismatches were removed. Forward and reverse sequence reads were then merged and trimmed with sequences shorter than $250 \mathrm{bp}$ discarded. Further sequence processing was executed with the referencebased OTU clustering module using the SILVA $16 \mathrm{~S}$ v 128 97\% database and the following setting: similarity threshold of $97 \%$, minimum occurrence of 2 , chimera crossover cost at 3, and maximum unaligned end mismatches at 5 . Raw sequence reads were deposited at the sequence read archive database (SRA) of NCBI under accession numbers SAMN14939471-SAMN14939499.
2.5 Identification of sources contributing to the indoor microbiomes

The contributions of potential microbial sources to the indoor microbiomes were determined with the SourceTracker software package (Knights et al., 2011), which estimates the proportion of the indoor microbiome derived from each of the potential sources using a Bayesian approach. Human skin and oral microbiota were chosen as the potential sources to be evaluated by SourceTracker analysis. Human skin and oral microbiota sequence data used in this study were retrieved from the Earth Microbiome Project and the European Nucleotide Archive (ENA), including 594 samples from the human skin (accession number ERR1867196-ERR1867464 and ERR1867837-ERR1868161) and 326 samples from the human mouth (accession number ERR1868427ERR1868749). These skin and oral samples were processed with the same primer sets used in this study $(515 \mathrm{~F}$ and $806 \mathrm{R})$, which facilitated sequence analysis. When a portion of the indoor microbiome did not match either of the potential sources, i.e. human skin or oral microbiota, this portion of the indoor microbiome would be assigned as being contributed from unknown sources.

\section{Results and discussion}

The indoor microbiome could be influenced by various factors, including occupant activities, design features, and management practices. The opening of new Building $\mathrm{M}$ on campus provided a rare opportunity to investigate the indoor microbiome when the impact of occupant activities was at a minimum. In contrast, Building $\mathrm{T}$, as another building on campus with identical design features and management practices, had been in full operation for six years. Thus, the comparisons between Buildings $\mathrm{M}$ and $\mathrm{T}$ could readily illustrate the impact of human occupancy on the indoor microbiomes.

\subsection{Microbial abundance in the indoor environment}

Heterotrophic plate count (HPC) was used as a measure of microbial abundance at 25 indoor surface sites in both Buildings M and T. HPC analysis showed that culturable heterotrophic bacteria were present at an average concentration of $18 \pm 19 \mathrm{CFU} / \mathrm{ft}^{2}$ in Building M (Fig. 1). In comparison, the abundance of culturable heterotrophic bacteria was $207 \pm 210 \mathrm{CFU} / \mathrm{ft}^{2}$ in Building T (Fig. 1). Despite considerable variations in HPC results, microbial abundance was significantly greater in Building $T$ than in Building $\mathrm{M}$, suggesting a potential correlation between increased occupant activities and greater levels of microbial presence in the indoor environment. 


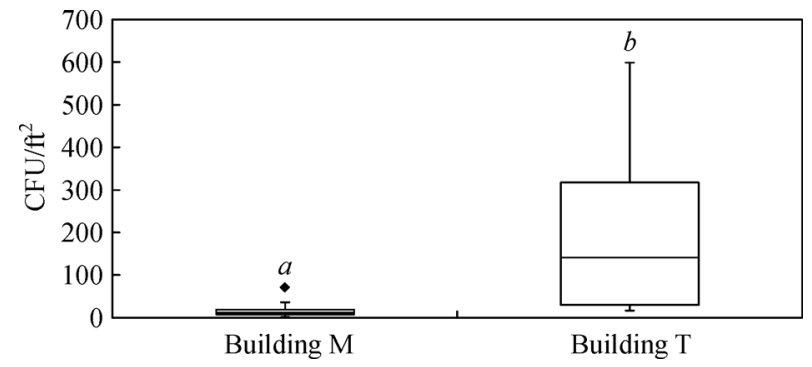

Fig. 1 HPC analysis of microbial abundance in the indoor environments with the box plots showing data from 25 surface samples in each building and the symbol " The means are not significantly different from each other in boxes labeled with the same italicized lowercase letters (Student's $t$-test, $p<0.05)$.

\subsection{Structure of indoor microbiomes}

Since HPC captured only a small subset of the indoor microbiome that could grow on a specific solid medium, more comprehensive profiles of the indoor microbiome were achieved with high-throughput sequencing of bacterial 16S rRNA gene amplicon libraries constructed for the same indoor surface samples from both buildings, resulting in a total of 13348 bacterial operational taxonomic units (OTUs) with $>97 \%$ sequence similarity, representing $88 \%$ of all clean reads.

To compare the structure of the indoor microbiomes between Buildings $\mathrm{T}$ and $\mathrm{M}$, results from 16S rRNA gene amplicon library sequencing of indoor surface samples were examined by principle coordinated analysis (PCoA) with weighted UniFrac distance (Fig. 2). It is evident that Buildings $\mathrm{M}$ and $\mathrm{T}$ accommodated distinct indoor microbiomes, as the microbial communities from the two buildings formed two distant clusters. It is noted that the

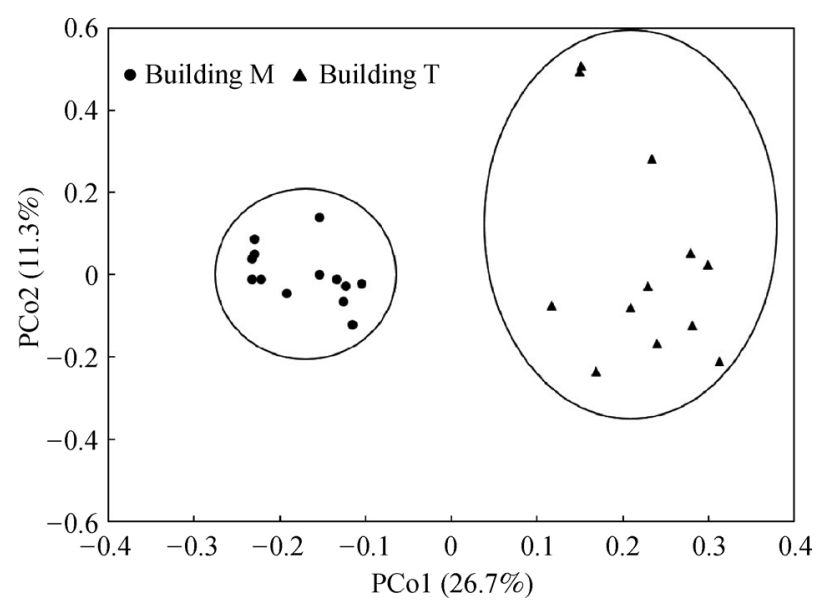

Fig. 2 Weighted principle coordinates analysis (PCoA) of the indoor microbiomes in Buildings $\mathrm{M}$ and T. Data points represent microbial community composition in indoor surface samples of Buildings $\mathrm{M}$ and $\mathrm{T}$ defined according to OTUs. cluster formed by indoor surface samples from Building $\mathrm{T}$ was much more scattered than that from Building $M$ (Fig. 2), suggesting the greater heterogeneity in the indoor microbiome of Building $\mathrm{T}$ than that of Building $\mathrm{M}$. One major difference between Buildings $\mathrm{M}$ and $\mathrm{T}$ was the level of human occupancy at the time of sampling. Building M was more homogenous in terms of occupancy because all rooms in the building had similarly low levels of occupancy as a new building with minimal use. In contrast, the levels of occupancy differed considerably between rooms in Building $T$, which had been subjected to extensive use for six years. Thus, the linkage between occupancy and indoor microbiome needed further investigation.

Analysis of similarities (ANOSIM) was conducted for the 16S rRNA gene sequences from the indoor surface samples to further illustrate the differences in the indoor microbiomes between the two buildings (Fig. 3). ANOSIM results $(R=0.597$ and $P=0.001)$ indicated that the dissimilarity in the indoor microbiomes between Buildings $\mathrm{M}$ and $\mathrm{T}$ was much greater than the dissimilarity within either Building M or T (Fig. 3), which is consistent with results from PCoA showing two distinct clusters between Buildings M and T (Fig. 2). ANOSIM further revealed that the dissimilarity in the indoor microbiomes within Building $\mathrm{T}$ was considerably higher than that within Building $\mathrm{M}$ (Fig. 3), in line with the greater indoor microbial heterogeneity identified by $\mathrm{PCoA}$ in Building $\mathrm{T}$ than in Building M (Fig. 2).

\subsection{Composition of indoor microbiomes}

To identify the microbial populations potentially contributing to the differences in the indoor microbiomes between Buildings $\mathrm{M}$ and $\mathrm{T}$, the composition of the indoor surface microbiomes was examined in more detail.

\subsubsection{Composition at phylum level}

Sequence analysis identified 44 and 55 bacterial phyla in

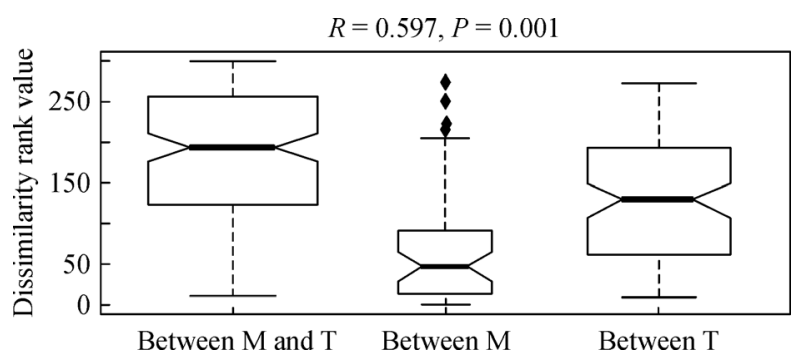

Fig. 3 Dissimilarity in indoor microbiome composition between and within Buildings $\mathrm{M}$ and $\mathrm{T}$ according to analysis of similarity (ANOSIM) with the box plots showing dissimilarity rank distribution sand the symbol " $\bullet$ indicating an outlier. The microbial community composition of indoor surface samples is defined according to OTUs. 
Buildings $\mathrm{M}$ and $\mathrm{T}$, respectively, indicating the greater population richness in Building $\mathrm{T}$. The most abundant phylum in Building $\mathrm{T}$ was Proteobacteria, representing $39 \%$ of the sequences (Fig. 4), which was followed by Actinobacteria and Firmicutes, accounting for $25 \%$ and $21 \%$ of the indoor microbial sequences, respectively (Fig. 4). Other significant populations in Building $\mathrm{T}$ included Cyanobacteria, Bacteroidetes, and Fusobacteria, with no other bacterial phyla representing $>1 \%$ of the indoor microbiome (Fig. 4).

For Building M, Proteobacteria was the most abundant phylum as well, accounting for $57 \%$ of the sequences, which however was much greater than its abundance in Building T (Fig. 4). Similar to Building T, Firmicutes, Actinobacteria, Bacteroidetes, and Cyanobacteria represented other dominant populations at the phylum level in Building $\mathrm{M}$, with relative abundance of $16 \%, 10 \%, 6 \%$, and $5 \%$, respectively. The only other phylum in Building $\mathrm{M}$ with relative abundance $>1 \%$ was Chloroflexi, which in comparison accounted for $<0.1 \%$ of the sequences in Building T (Fig. 4).

While the phylum-level composition of the indoor microbiomes differed slightly between Buildings $\mathrm{M}$ and T, the most abundant populations were identical in the two buildings, including Proteobacteria, Firmicutes, Actinobacteria, Bacteroidetes, and Cyanobacteria. These results are consistent with previous indoor microbiome studies, where populations associated with these phyla have been frequently identified in a variety of indoor environments (Hewitt et al., 2012; Kembel et al., 2012; Kembel et al., 2014; Hoisington et al., 2016).

\subsubsection{Composition at genus level}

The indoor microbiomes exhibited more distinctions at the genus level between Buildings $\mathrm{M}$ and $\mathrm{T}$. The most frequently detected genus in Building $\mathrm{M}$ was Rhizobium, accounting for $18 \%$ of the sequences on average (Fig. 5). In contrast, Rhizobium was identified with a much lower relative abundance in Building $\mathrm{T}$, averaging 3.7\% only. Given that Rhizobium populations are known to be soil bacteria specialized in nitrogen fixation (Sawada et al., 2003), the higher abundance of Rhizobium found in Building $\mathrm{M}$ is indicative of a greater exposure to the outdoor environment, which is consistent with the fact that construction of Building $\mathrm{M}$ was only recently completed.

Stenotrophomonas was another bacterial genus frequently detected in Building $\mathrm{M}$ at an average abundance of $12.7 \%$, accounting for more than $50 \%$ of the sequences at two of the sampling locations in Building M (Fig. 5). In comparison, Stenotrophomonas was present at a significantly lower relative abundance in Building $\mathrm{T}$, only contributing $1.0 \%$ on average to the indoor microbiome. Populations of Stenotrophomonas are found throughout the environment, particularly in close association with plants (Ryan et al., 2009). Thus, the higher abundance of these bacteria in Building $M$ could again be attributed to greater influences from the outdoor environment as a result of recent construction activities in the new building.

An alarming finding in Building T was the abundance of Staphylococcus strains, representing on average $8.5 \%$ of the indoor microbiome, which was significantly higher than the relative abundance of Staphylococcus in Building

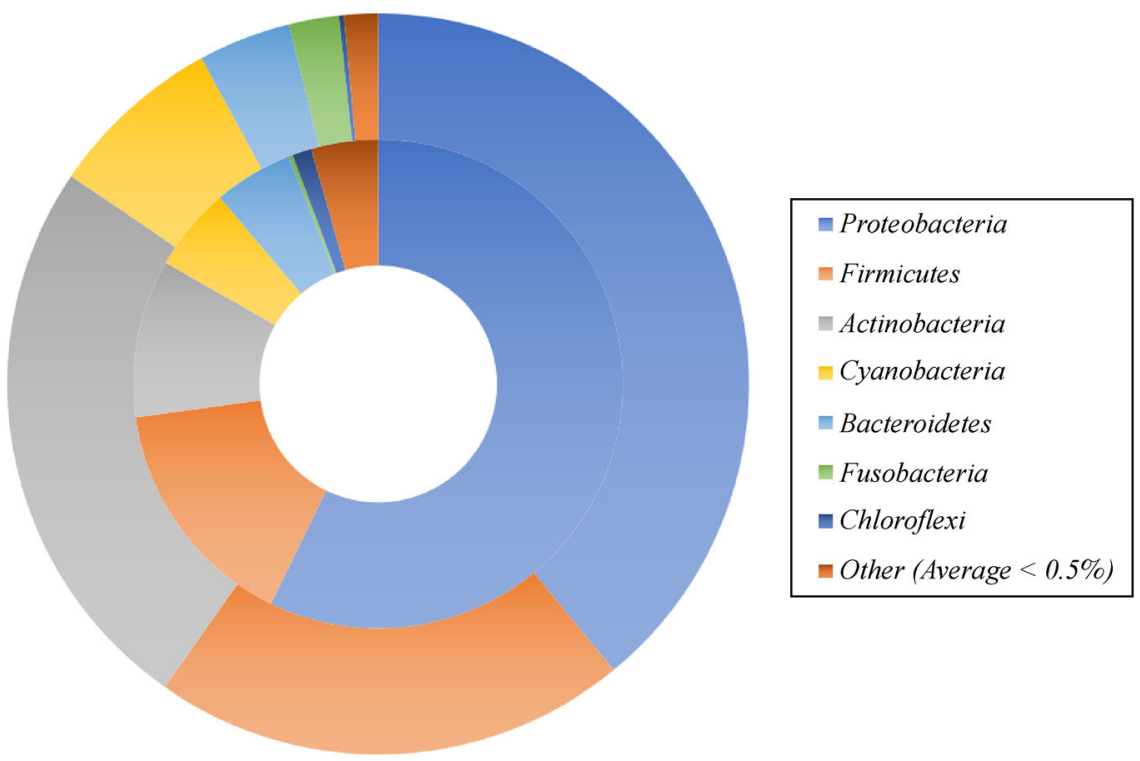

Fig. 4 Comparison of indoor microbiome composition between Buildings $\mathrm{T}$ and $\mathrm{M}$ at the phylum level. Outer ring: Building T; inner ring: Building $\mathrm{M}$. 


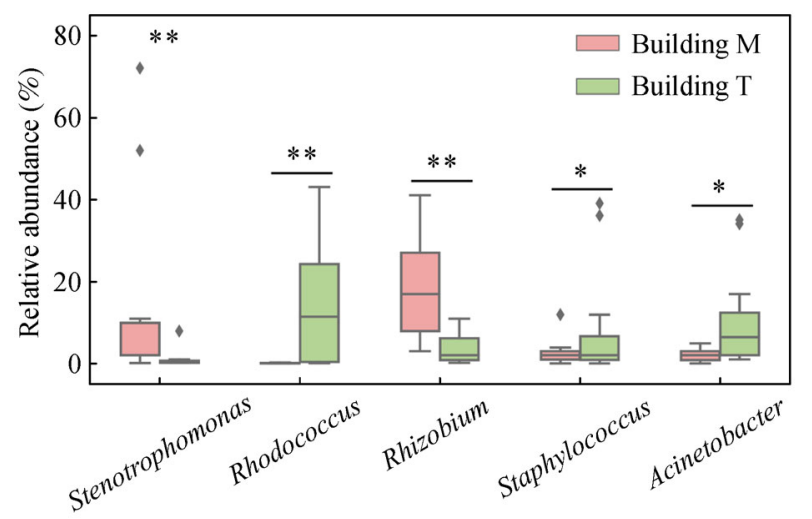

Fig. 5 The relative abundance of bacterial genera with average relative abundance greater than 5\% in indoor microbiomes. The asterisks on top of box plots show the level of significance in the difference between Buildings $\mathrm{T}$ and $\mathrm{M}$, with $*$ indicating $p<0.05$ and $* *$ indicating $p<0.01$ according to the Wilcoxon rank-sum test. The symbol "»" indicates an outlier.

M, averaging $2.5 \%$ (Fig. 5). It is known that members of Staphylococcus, considered opportunistic pathogens, frequently colonize the skin and mucous membranes of warm-blooded animals, including humans (Baird-Parker, 1990). Given that humans are natural hosts of Staphylococcus, the greater presence of Staphylococcus populations in Building T could be attributed to higher levels of human activities in Building $\mathrm{T}$ with six years of operation than those in new Building $\mathrm{M}$, which was yet to experience substantial human occupancy.

Other abundant microbial inhabitants of Building $\mathrm{T}$ included members of Acinetobacter, accounting for $10.7 \%$ of the indoor microbiome (Fig. 5). In comparison, the relative abundance of Acinetobacter averaged only $2.1 \%$ in Building M, which was significantly lower than that in Building T. Similar to Staphylococcus, Acinetobacter populations are known to be opportunistic pathogens and constituents of the normal human skin microflora (Towner, 2009). Thus, the greater abundance of Acinetobacter in Building $\mathrm{T}$, where human occupancy was much more extensive, again points to the potential importance of human activities in shaping the indoor microbiome.

Besides Rhizobium, Stenotrophomonas, Acinetobacter, and Staphylococcus, the only other bacterial genus with the average relative abundance greater than $5 \%$ in either building was Rhodococcus. Indeed, with its relative abundance averaging $14.8 \%$, Rhodococcus was the most abundant genus in Building T. Interestingly, the presence of Rhodococcus in Building M, however, was minimal, with less than $0.1 \%$ of the indoor microbiome identified as Rhodococcus (Fig. 5). This sharp contrast between Buildings $\mathrm{T}$ and $\mathrm{M}$ was unexpected. Because Rhodococcus strains are known to be broadly distributed in the environment (Bell et al., 1998), it would be more reasonable to expect greater abundance of Rhodococcus in Building $\mathrm{M}$ instead of Building $\mathrm{T}$, as observed for Rhizobium and Stenotrophomonas, both having broad environmental distributions and consequently greater abundance in Building $\mathrm{M}$ rather than Building T (Fig. 5). Further examination of the Rhodococcus sequences was needed to shed more light on the unexpected abundance of Rhodococcus in Building T.

\subsection{Phylogenetic analysis of abundant OTUs}

The sequences of the abundant genera in the indoor microbiomes, i.e. relative abundance $>5.0 \%$ in either Building $\mathrm{T}$ or $\mathrm{M}$, were grouped into OTUs with $>97 \%$ sequence similarity (Fig. 6). One important distinction between Buildings $\mathrm{M}$ and $\mathrm{T}$ was the significantly higher abundance of two genera, Rhizobium and Stenotrophomonas, in Building M (Fig. 5). Rhizobium was represented exclusively by OTU2, which is most closely related to Rhizobium leguminosarum (Fig. 6). Similar to all rhizobia, $R$. leguminosarum is an obligate symbiont of legumes carrying out nitrogen fixation in root nodules (MassonBoivin et al., 2009). The genus Stenotrophomonas comprised OTU7, which is most closely related to Stenotrophomonas maltophilia (Fig. 6). S. maltophilia has broad distribution in the natural environment, particularly abundant in the plant rhizosphere with ecological functions in nutrient cycling (Hayward et al., 2010). The findings that soil is the natural habitat of both OTU2 and OTU7 suggest that populations of Rhizobium and Stenotrophomonas identified in Buildings $\mathrm{M}$ and $\mathrm{T}$ were likely originated from the soil. It could be reasoned that, as a new construction, Building $\mathrm{M}$ had greater resemblance to the outdoor environment and subsequently greater abundance of soil-associated microbial populations such as Rhizobium and Stenotrophomonas. It should be noted that $S$. maltophilia has been frequently isolated from clinical environments as an emerging opportunistic pathogen (An and Berg, 2018). It is unlikely that rigorous microbial control practices in these clinical environments 


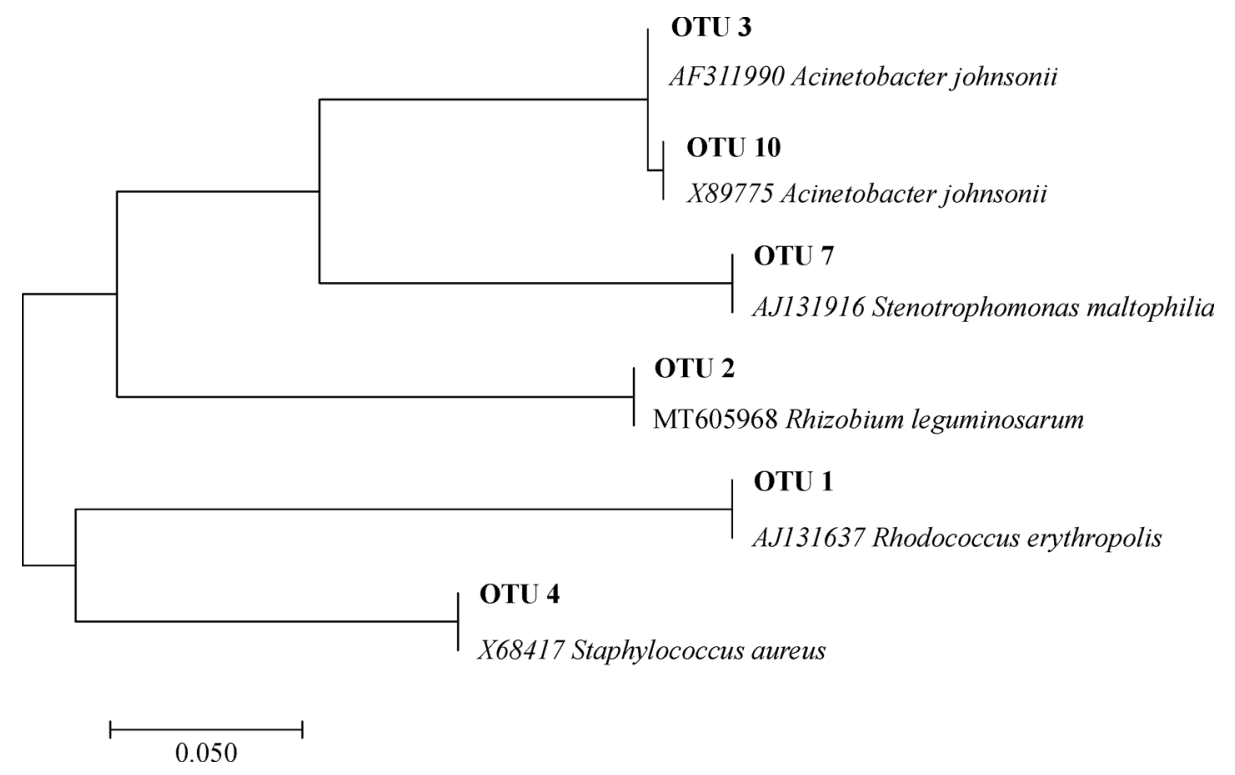

Fig. 6 Maximum-likelihood tree showing abundant 16S rRNA gene sequences in indoor microbiomes (average relative abundance $>5 \%$ ) in relation to sequences of closest reference strains, which were obtained by SeqMatch as the first perfect match (similarity score $=$ 1.0) in the Ribosomal Database Project (RDP). GenBank accession numbers of the reference strains are shown together with the name of the strains. The scale bar represents the number of substitutions per sequence position.

would promote the introduction of soil microorganisms from the outdoor environment. Instead, this might be the result of the exceptional metabolic persistence and versatility that enable the adaptation of $S$. maltophilia to these unique environments (Ryan et al., 2009).

While new Building $M$ was predominated by soil microbial populations represented by Rhizobium and Stenotrophomonas, Building $\mathrm{T}$ was characterized by the significantly higher abundance of Acinetobacter, Staphylococcus, and Rhodococcus (Fig. 5). Sequences of Acinetobacter were grouped into two OTUs, i.e. OTU3 and OTU10; however, both OTUs are closely related to Acinetobacter johnsonii (Fig. 6). Previous studies have frequently identified humans and human-impacted habitats as reservoirs of $A$. johnsonii and other Acinetobacter strains (Al Atrouni et al., 2016). The abundance of $A$. johnsonii in Building $\mathrm{T}$ might also be attributable to its ability to survive dry conditions for prolonged periods (Hirai, 1991). Phylogenetics analysis revealed that sequences of Staphylococcus formed OTU4, most closely related to Staphylococcus aureus (Fig. 6). It has been recognized that $S$. aureus is an endogenous pathogen which colonizes the human nose as its preferred reservoir in $30 \%$ of the human population (Krismer et al., 2017). Thus, the higher abundance of human-associated Acinetobacter and Staphylococcus in Building T is consistent with more extensive human occupancy in Building $\mathrm{T}$ than in Building M.

Rhodococcus was another bacterial genus highly abundant in Building $\mathrm{T}$ but not in Building $\mathrm{M}$ (Fig. 5). Phylogenetic analysis indicates that the Rhodococcus sequences in the indoor microbiomes were represented by Rhodococcus erythropolis (Fig. 6), which is broadly distributed in diverse natural environments (de Carvalho and da Fonseca, 2005). R. erythropolis is known to have hydrophobic cells with the ability to attach to surfaces (Rodrigues and de Carvalho, 2015), providing a potential mechanism contributing to the abundance of $R$. erythropolis on indoor surfaces in Building T. However, it could not explain the significantly lower abundance of $R$. erythropolis in Building M (Fig. 5). If outdoor environment was the primary source of indoor $R$. erythropolis, its abundance in Building $M$ would be expected to be higher given the greater influence of the outdoor environment on Building $M$ as a new construction. An alternative postulation is that $R$. erythropolis in the indoor environment could have human origins, which is supported by reports that $R$. erythropolis could colonize humans along with other endogenous pathogens such as staphylococci (Graham et al., 2007). This would explain the higher abundance of $R$. erythropolis in Building $\mathrm{T}$ due to its longer history of human occupancy than Building $\mathrm{M}$. Further studies are needed to assess the importance of humans as the reservoir of $R$. erythropolis.

\subsection{Indoor microbiome source identification}

Given the significance of human occupancy in shaping the indoor microbiomes as revealed by phylogenetic analysis, SourceTracker was used to quantify the contribution of human-associated microorganisms as sources to the indoor microbiomes (Knights et al., 2011). The dispersal of human-associated microorganisms in the indoor environment occurs primarily via direct surface contact, desqua- 

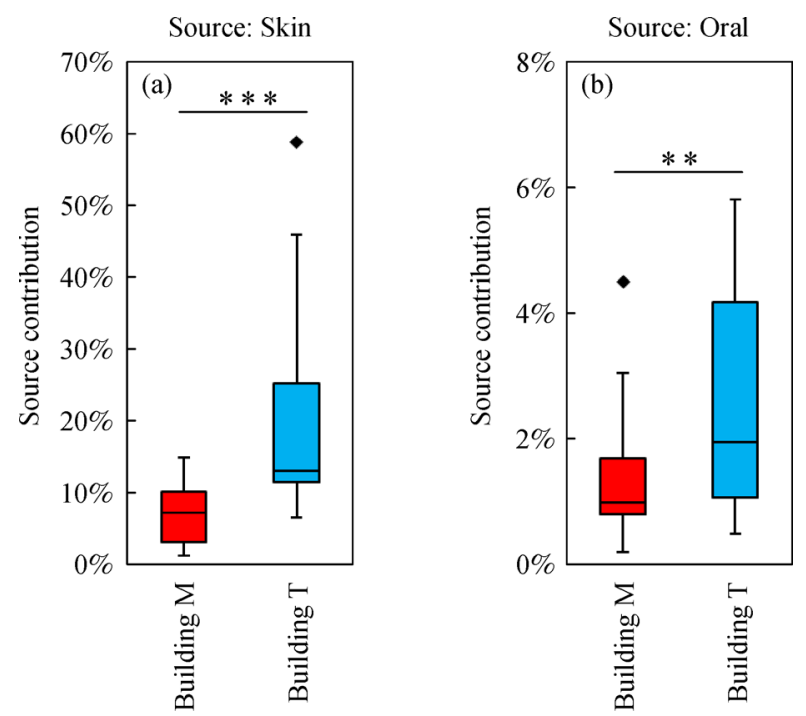

Fig. 7 Percentage of indoor microbiomes contributed by human skin (a) and oral (b) sources in Buildings M and T. The asterisks on top of box plots show the level of significance in the difference between Buildings $\mathrm{T}$ and $\mathrm{M}$, with * indicating $p<0.05$ and $* *$ indicating $p<0.01$ according to the Student $t$-test. The symbol "»" indicates an outlier.

mation, or emission of bioaerosols and particulates from human breath (Lax et al., 2014; Meadow et al., 2014). Therefore, human skin and oral microbiota were chosen as the sources for SourceTracker analysis.

It was evident that a significantly greater portion of the indoor microbiome in Building $\mathrm{T}$ originated from humanassociated microorganisms than in Building M (Fig. 7). Specifically, human skin microbiota represented a median contribution of $13.4 \%$ to the indoor microbiome in Building T, which was significantly higher than the median contribution of $6.2 \%$ in Building M (Fig. 7(a)). Compared with human skin microbiota, the contribution from human oral microbiota to the indoor microbiomes was much smaller, with the median at $2.8 \%$ and $0.7 \%$ in Buildings $\mathrm{T}$ and $M$, respectively (Fig. 7(b)). Similar to the skin microbiota, the oral microbiota contributed to the indoor microbiome in Building $\mathrm{T}$ at significantly higher levels than in Building M. These results show that the impact of human-associated microorganisms was significantly greater on the indoor microbiome in Building $\mathrm{T}$ than in Building $\mathrm{M}$, which is consistent with more extensive human occupancy in Building $\mathrm{T}$ than Building $\mathrm{M}$.

It is noted that results from SourceTracker analysis exhibited large variations (Fig. 7). For example, the median contribution of human skin microbiota to the indoor microbiomes in Building $\mathrm{T}$ was $13.4 \%$, with the maximum reaching $54.3 \%$ (Fig. 7(a)). Thus, it could be hypothesized that some areas in the indoor environment had much greater influence from the occupants and associated microbiota than others. To test this hypothesis, the sampling sites in the two buildings were divided into two groups - high-occupancy areas, including classrooms and public areas (lobbies), and low-occupancy areas such as offices and research laboratories. In Building $T$, both human skin and oral microbiota sources were found to contribute to indoor microbiomes at significantly greater levels in high-occupancy areas than in low-occupancy areas (Fig. 8), confirming the important impact of human occupancy on the indoor microbiome. In contrast, in Building $\mathrm{M}$, no significant differences were detected in human contribution to the indoor microbiomes between high-occupancy areas and low-occupancy areas (Fig. 8), which is likely due to the lack of extensive human occupancy even in the high-occupancy areas of Building $M$. These results again highlight the significance of human occupancy in shaping the indoor microbiome, which has been suggested in studies of indoor microbiomes in other types of built environments (Gilbert and Stephens, 2018).

\section{Conclusions}

Comparisons were made between the indoor microbiomes of two multifunctional university buildings, of which one was new with minimal human occupancy while the other was older with six years of occupancy. Higher levels of human occupancy in the older building were shown to result in greater microbial abundance in the indoor environment and greater proportion of the indoor microbiome to be contributed from human-associated microbiota, particularly the skin microbiota. It was further revealed that human-associated microbiota had greater influence on the indoor microbiome in areas of high occupancy than areas of low occupancy in the same building. Consistent with minimal impact from human occupancy in a new construction, the indoor microbiomes 

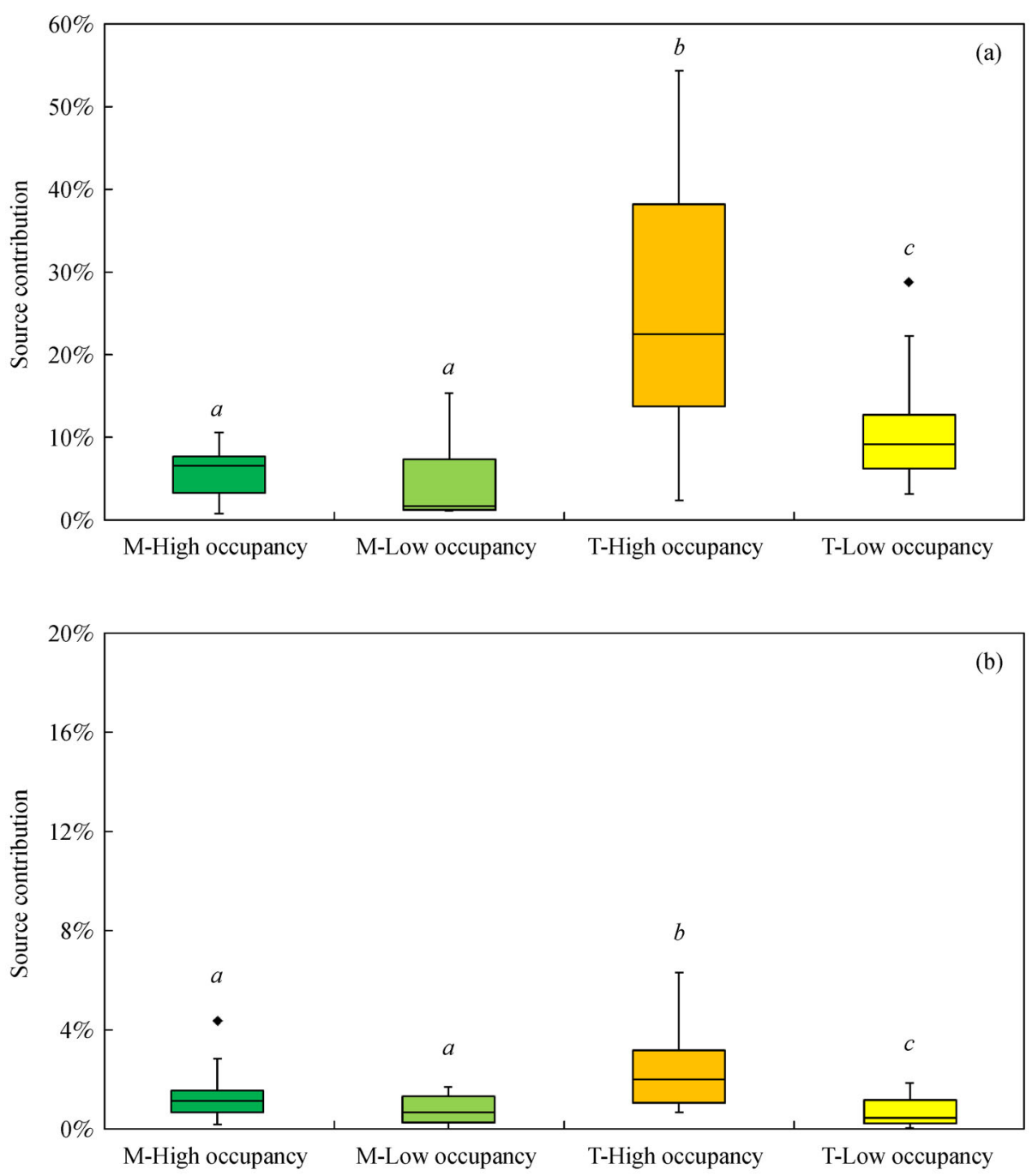

Fig. 8 Percentage of indoor microbiomes contributed by human skin (a) and oral (b) sources in high- and low-occupancy areas of Buildings $\mathrm{M}$ and T. M-Building M; T-Building T. High occupancy-samples collected from classrooms and lobbies; lowoccupancy - samples taken from offices and research laboratories. The means are not significantly different from each other in boxes labeled with the same italicized lowercase letters (Student's $t$-test, $p<0.05$ ). The symbol " $"$ indicates an outlier.

in the new building exhibited significantly lower influence from human-associated microbiota than in the older building, with microbial taxa originating from soil and plants representing the dominant constituents of the indoor microbiome. In contrast, abundant microbial taxa in the older building were represented by constituents of the human microbiota, likely derived from occupants. These findings provide insights into the processes shaping the indoor microbiomes for the development of effective strategies to manage the microbial exposure of occupants in the built environment.

Acknowledgements This work was supported in part by US National Science Foundation awards 1952140 and 2026719. Any opinions, findings, recommendations, and conclusions in this paper are those of the authors, and do not necessarily reflect the views of NSF and the University of Tennessee, Knoxville of USA.

\section{References}

Adams R I, Bateman A C, Bik H M, Meadow J F (2015). Microbiota of the indoor environment: A meta-analysis. Microbiome, 3: 49

Al Atrouni A, Joly-Guillou M L, Hamze M, Kempf M (2016). Reservoirs of non-baumannii Acinetobacter species. Frontiers in Microbiology, 7: 49

Allen J G, Marr L C (2020). Recognizing and controlling airborne transmission of SARS-CoV-2 in indoor environments. Indoor Air, 30 (4): 557-558

An S Q, Berg G (2018). Stenotrophomonas maltophilia. Trends in Microbiology, 26(7): 637-638

Baird - Parker A C (1990). The staphylococci: An introduction. Journal of Applied Bacteriology, 69: 1S-8S

Bell K S, Philp J C, Aw D W J, Christofi N (1998). The genus Rhodococcus. Journal of Applied Microbiology, 85(2): 195-210 
Ben Maamar S, Hu J, Hartmann E M (2020). Implications of indoor microbial ecology and evolution on antibiotic resistance. Journal of Exposure Science \& Environmental Epidemiology, 30(1): 1-15

de Carvalho C C C R, da Fonseca M M R (2005). The remarkable Rhodococcus erythropolis. Applied Microbiology and Biotechnology, 67(6): 715-726

Gilbert J A, Stephens B (2018). Microbiology of the built environment. Nature Reviews. Microbiology, 16(11): 661-670

Graham J E, Moore J E, Jiru X U, Moore J E, Goodall E A, Dooley J S, Hayes V E, Dartt D A, Downes C S, Moore T C (2007). Ocular pathogen or commensal: a PCR-based study of surface bacterial flora in normal and dry eyes. Investigative Ophthalmology \& Visual Science, 48(12): 5616-5623

Hayward A C, Fegan N, Fegan M, Stirling G R (2010). Stenotrophomonas and Lysobacter: Ubiquitous plant-associated gamma-proteobacteria of developing significance in applied microbiology. Journal of Applied Microbiology, 108(3): 756-770

Hewitt K M, Gerba C P, Maxwell S L, Kelley S T (2012). Office space bacterial abundance and diversity in three metropolitan areas. PLoS One, 7(5): e37849

Hirai Y (1991). Survival of bacteria under dry conditions; from a viewpoint of nosocomial infection. Journal of Hospital Infection, 19 (3): 191-200

Hoisington A, Maestre J P, Kinney K A, Siegel J A (2016). Characterizing the bacterial communities in retail stores in the United States. Indoor Air, 26(6): 857-868

Kembel S W, Jones E, Kline J, Northcutt D, Stenson J, Womack A M, Bohannan B J, Brown G Z, Green J L (2012). Architectural design influences the diversity and structure of the built environment microbiome. ISME Journal, 6(8): 1469-1479

Kembel S W, Meadow J F, O'Connor T K, Mhuireach G, Northcutt D, Kline J, Moriyama M, Brown G Z, Bohannan B J M, Green J L (2014). Architectural design drives the biogeography of indoor bacterial communities. PLoS One, 9(1): e87093

Klepeis N E, Nelson W C, Ott W R, Robinson J P, Tsang A M, Switzer P, Behar J V, Hern S C, Engelmann W H (2001). The National Human Activity Pattern Survey (NHAPS): a resource for assessing exposure to environmental pollutants. Journal of Exposure Science \& Environmental Epidemiology, 11(3): 231-252

Knights D, Kuczynski J, Charlson E S, Zaneveld J, Mozer M C, Collman R G, Bushman F D, Knight R, Kelley S T (2011). Bayesian community-wide culture-independent microbial source tracking. Nature Methods, 8(9): 761-763

Krismer B, Weidenmaier C, Zipperer A, Peschel A (2017). The commensal lifestyle of Staphylococcus aureus and its interactions with the nasal microbiota. Nature Reviews. Microbiology, 15(11): 675-687

Lax S, Gilbert J A (2015). Hospital-associated microbiota and implications for nosocomial infections. Trends in Molecular Medicine, 21(7): 427-432

Lax S, Nagler C R, Gilbert J A (2015). Our interface with the built environment: immunity and the indoor microbiota. Trends in Immunology, 36(3): 121-123
Lax S, Smith D P, Hampton-Marcell J, Owens S M, Handley K M, Scott N M, Gibbons S M, Larsen P, Shogan B D, Weiss S, Metcalf J L, Ursell L K, Vazquez-Baeza Y, Van Treuren W, Hasan N A, Gibson M K, Colwell R, Dantas G, Knight R, Gilbert J A (2014). Longitudinal analysis of microbial interaction between humans and the indoor environment. Science, 345(6200): 1048-1052

Masson-Boivin C, Giraud E, Perret X, Batut J (2009). Establishing nitrogen-fixing symbiosis with legumes: how many rhizobium recipes? Trends in Microbiology, 17(10): 458-466

Meadow J F, Altrichter A E, Kembel S W, Moriyama M, O’Connor T K, Womack A M, Brown G Z, Green J L, Bohannan B J (2014). Bacterial communities on classroom surfaces vary with human contact. Microbiome, 2: 7

Reasoner D J (2004). Heterotrophic plate count methodology in the United States. International Journal of Food Microbiology, 92(3): 307-315

Rodrigues C J C, de Carvalho C C C R (2015). Rhodococcus erythropolis cells adapt their fatty acid composition during biofilm formation on metallic and non-metallic surfaces. FEMS Microbiology Ecology, 91(12): fiv135

Rose L, Jensen B, Peterson A, Banerjee S N, Arduino M J (2004). Swab materials and Bacillus anthracis spore recovery from nonporous surfaces. Emerging Infectious Diseases, 10(6): 1023-1029

Ryan R P, Monchy S, Cardinale M, Taghavi S, Crossman L, Avison M B, Berg G, Van Der Lelie D, Dow J M (2009). The versatility and adaptation of bacteria from the genus Stenotrophomonas. Nature Reviews. Microbiology, 7(7): 514-525

Sawada H, Kuykendall L D, Young J M (2003). Changing concepts in the systematics of bacterial nitrogen-fixing legume symbionts. Journal of General and Applied Microbiology, 49(3): 155-179

Sbihi H, Boutin R C, Cutler C, Suen M, Finlay B B, Turvey S E (2019). Thinking bigger: How early-life environmental exposures shape the gut microbiome and influence the development of asthma and allergic disease. Allergy, 74(11): 2103-2115

Schweizer C, Edwards R D, Bayer-Oglesby L, Gauderman W J, Ilacqua V, Juhani MJantunen H K, Lai M, Nieuwenhuijsen N, Künzli (2007). Indoor time-microenvironment-activity patterns in seven regions of Europe. Journal of Exposure Science \& Environmental Epidemiology, 17(2): 170-181

Song L, Wang C, Wang Y (2020). Optimized determination of airborne tetracycline resistance genes in laboratory atmosphere. Frontiers of Environmental Science and Engineering, 14(6): 95

Towner K J (2009). Acinetobacter: an old friend, but a new enemy. Journal of Hospital Infection, 73(4): 355-363

Treu L, Kougias P G, de Diego-Díaz B, Campanaro S, Bassani I, Fernández-Rodríguez J, Angelidaki I (2018). Two-year microbial adaptation during hydrogen-mediated biogas upgrading process in a serial reactor configuration. Bioresource Technology, 264: 140-147

US Census Bureau (2018). Classrooms More Racially and Ethnically Diverse. Suitland,: US Census Bureau

Wyckoff K N, Chen S, Steinman A J, He Q (2017). Impact of roadway stormwater runoff on microbial contamination in the receiving stream. Journal of Environmental Quality, 46(5): 1065-1071 